\title{
INFLUENCE OF RECIPROCATING COMPRESSOR STRUCTURE ON TORQUE DIAGRAM
}

\author{
A.A. KOTLOV ${ }^{1}$, L. G. KUZNETSOV ${ }^{2}$ \& A.V. BURAKOV ${ }^{3}$ \\ ${ }^{I}$ National Technology Initiative Center for Advanced Manufacturing Technologies based on the Institute of Advanced \\ Manufacturing Technologies of Peter the Great St. Petersburg Polytechnic University Polytechnicheskaya, 29, \\ St.Petersburg, 195251, Russia \\ ${ }^{2,3}$ JSC "Compressor" 64, Bolshoy Sampsonievsky Pr., St. Petersburg 194044, Russia
}

\begin{abstract}
A variable summand of the rotary mechanism reaction torque, in particular that of the reciprocating compressors, is one of the main sources of their low-frequency vibration. Being solved in this study is a problem of providing the constantsign transmitted torque of the multistage reciprocating compressor in order to decrease its vibration. The investigation results concerning the influence of different structural parameters on the torque diagram are given. The description of a software program developed specially to carry out this investigation is represented. The description of the structural solution is given aimed at obtaining the most uniform torque diagram which has a constant sign during a single revolution of the crankshaft.

KEYWORDS: Reciprocating Compressor, Torque, Vibration, Mathematical Modeling
\end{abstract}

Received: Jun 09, 2020; Accepted: Jun 29, 2020; Published: Sep 28, 2020; Paper Id.: IJMPERDJUN20201449

\section{INTRODUCTION}

It is widely known [1] that a variable summand of the rotary mechanism reaction torque, in particular that of the reciprocating compressors, is one of the main sources of their low-frequency vibration. The means of complete suppression of this vibration source, irrespective of the mechanism operation mode, is creation of a compensating moment in the transmission supports, providing the counter-rotation of rotors with a certain ratio of their inertia moments.

In the majority of structures the counter-rotation is provided by the gearwheel. At that, a necessity occurs to exclude the dynamic displacement of teeth, which decreases the transmission resource and invokes the increased noise. As for the reciprocating compressors, this requirement is met through constant-sign transmitted torque. I.e. the counter-torque at the compressor shaft and, consequently, the reaction torque equal thereto and applied to the housing, must not change its sign during the whole revolution and must be completely carried-over through the transmission with the external gearing [2].

The research object is the four-stage reciprocating compressor intended for use in the gas-transmission and gas-filling compressor stations. Figure 1 contains a general view and movement mechanism of the compressor under consideration. The given compressor is developed by Compressor JSC and made with a crankshaft vertical configuration [3]. The compressor is intended for compression of methane or natural gas to the final pressure of 24.6 MPa, with a short-term operation up to a pressure of $39.2 \mathrm{MPa}$. 


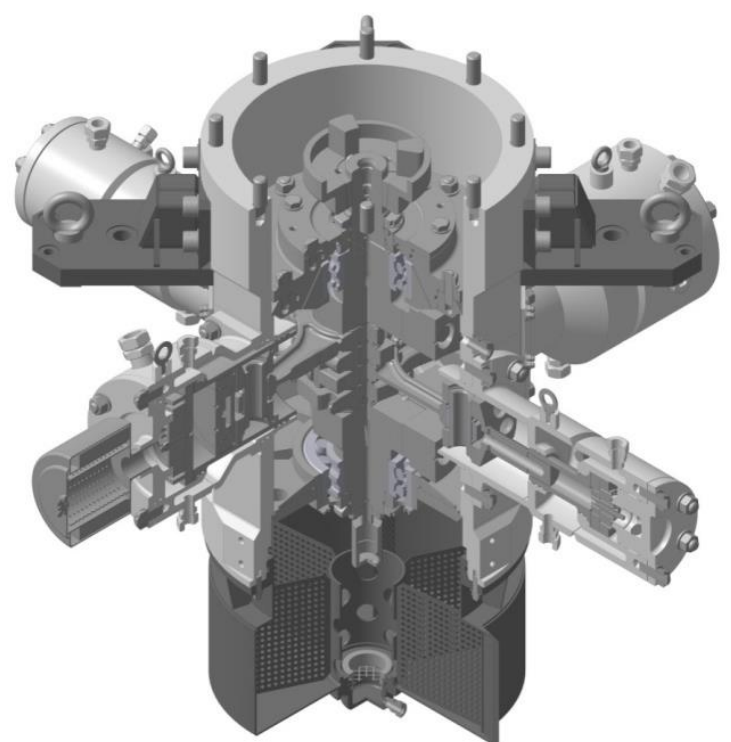

a) General View

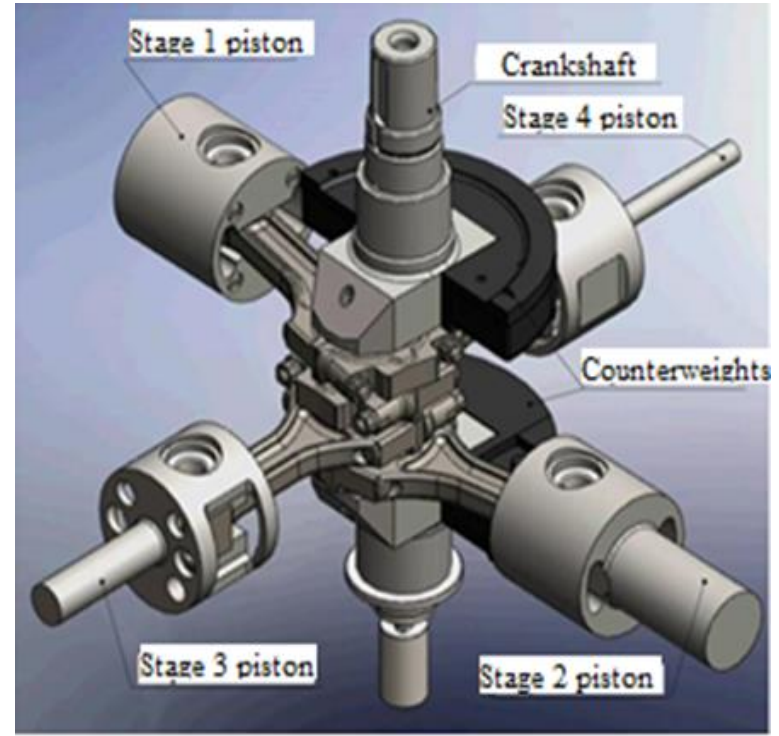

b) Motion Work

Figure 1: Scheme of Compressor with Vertical Shaft.

\section{CALCULATION METHOD}

Piston forces $\mathrm{P} \Sigma$ in the crankshaft mechanism are disintegrated into two components: a normal force to the cylinder axis $\mathrm{N}$ and a force acting along the piston rod Ppr (see Figure 2) [1, 4-7].

The normal force presses the piston to the cylinder wall in case of a closed-crankcase compressor, or presses the cross-head to parallels in case of an open-crankcase compressor. The force acting along the piston rod loads the crank pin of the shaft and is disintegrated into the radial component directed to the shaft axis Pr and the tangential component Pt (see Figure 2).

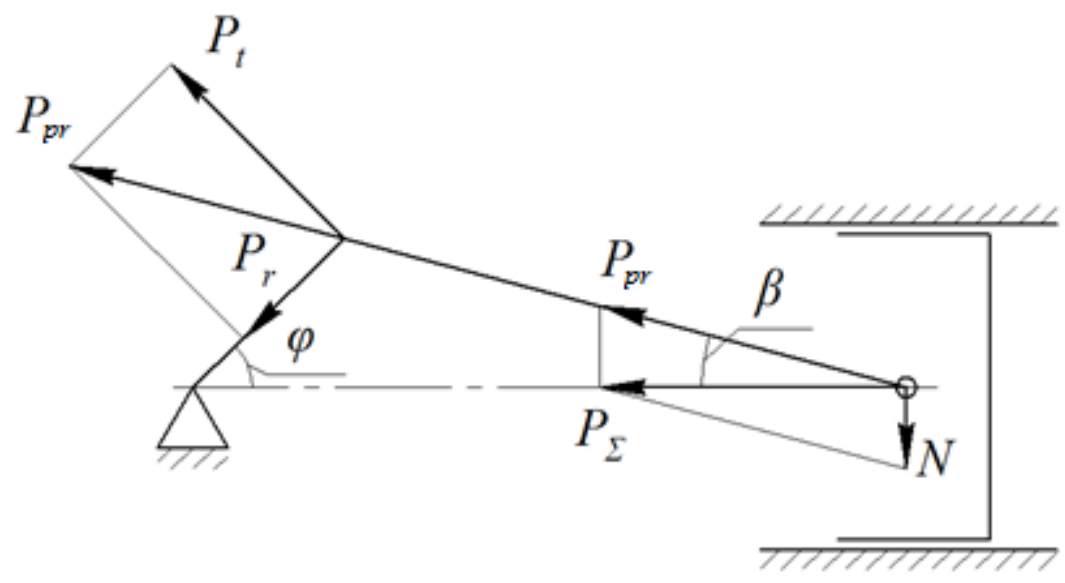

Figure 2: Diagram of Forces Action.

The force Pt applied to the crank pin on a radius $r$ creates a torque counteracting to the shaft rotation. This torque value is determined as per the formula $[1,5-8]$

$$
M_{t}=P_{t} r
$$


For multi-row compressors the torque shall be determined as a sum of torques in each row

$$
M_{t}=\sum P_{t i} r_{i}
$$

Besides, the friction force from rotating parts has an influence on the torque value. The friction force from rotating parts is considered as constant, so the torque created by this force will be constant and equal to

$$
M_{f}=(0,3 \div 0,4) \frac{N_{\text {ind }}}{2 \pi n_{0}}\left(\frac{1}{\eta_{m}}-1\right)
$$

Where Nind is indicated power, $\mathrm{n} 0$ is crankshaft speed, $\eta \mathrm{m}$ is mechanical efficiency $(\eta \mathrm{m}=0.8 \ldots 0.95)$.

The tangential force $\mathrm{Pt}$ is determined by formula

$$
P_{t}=P_{\Sigma} \frac{\sin (\varphi+\beta)}{\cos \beta}
$$

Total force is determined by formula

$$
P_{\Sigma}=P_{g}+I_{s}+F_{f}
$$

Where Pg is a force of gas action to the piston, Is is inertial forces of reciprocating masses, Ff is friction forces at reciprocating action.

The force $\mathrm{Pg}$ is calculated on the basis of the stage indicator diagram, which is obtained by mathematical modeling of the stage operating process $[7,9]$. The mathematical model is based on known models of the working process of reciprocating compressors [10-15].

The value Is is determined by formula

$$
I_{s}=m_{s} r \omega^{2}(\cos \varphi+\lambda \cos 2 \varphi)
$$

Where $\mathrm{ms}$ is mass of reciprocating parts, $\omega$ is angular speed, $\lambda$ is ratio between crank radius and piston rod length.

\section{CALCULATED ANALYSIS AND INITIAL DATA}

The torque character will change at different arrangement of cranks. Having plotted the total torque curves for different variants of cranks arrangement it is possible to achieve their most favorable position. R\&D Laboratory "Gas Dynamics of Turbomachines" SPbPU actively develops software products for solving various problems: for the design of reciprocating compressors $[3,7,9,10]$, for the design of rotary compressors [16, 17], as well as for the design of turbo compressors [18 30]. In order to analyze the influence of different factors on the torque diagram the software program is developed (see Figure 3). The calculation results are represented in numerical and graphical form and contain: power diagrams for each row of compressor; diagrams of tangential forces; diagram of total counter-torque, etc. 
The program allows making calculation for different structural design of the compressor. To this end, the row constructor is developed which makes it possible to take into account the mutual arrangement of cylinders and stages location by rows. Figure 4 shows a program window in the mode of the compressor row designing.

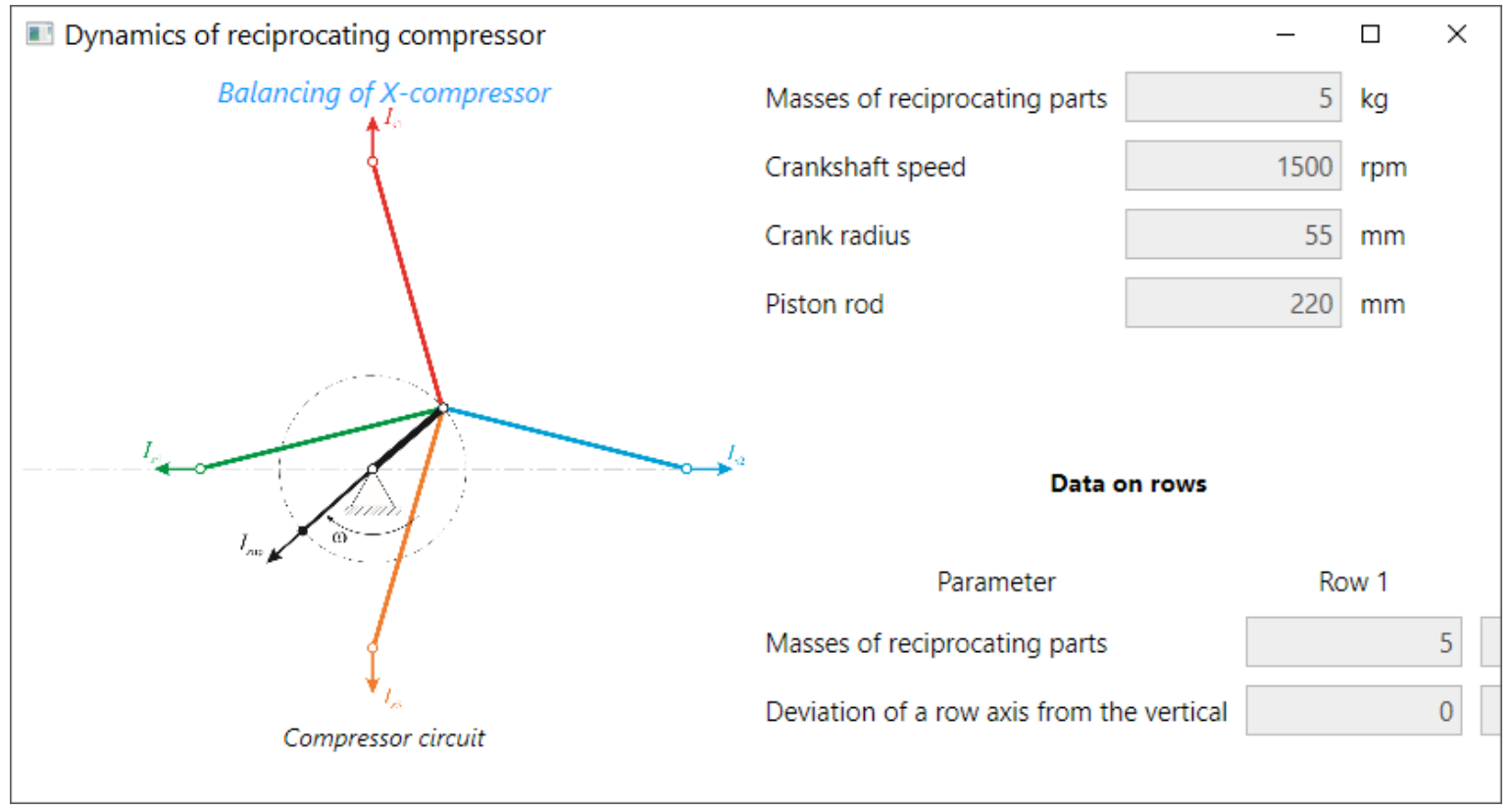

Figure 3: Program Window.

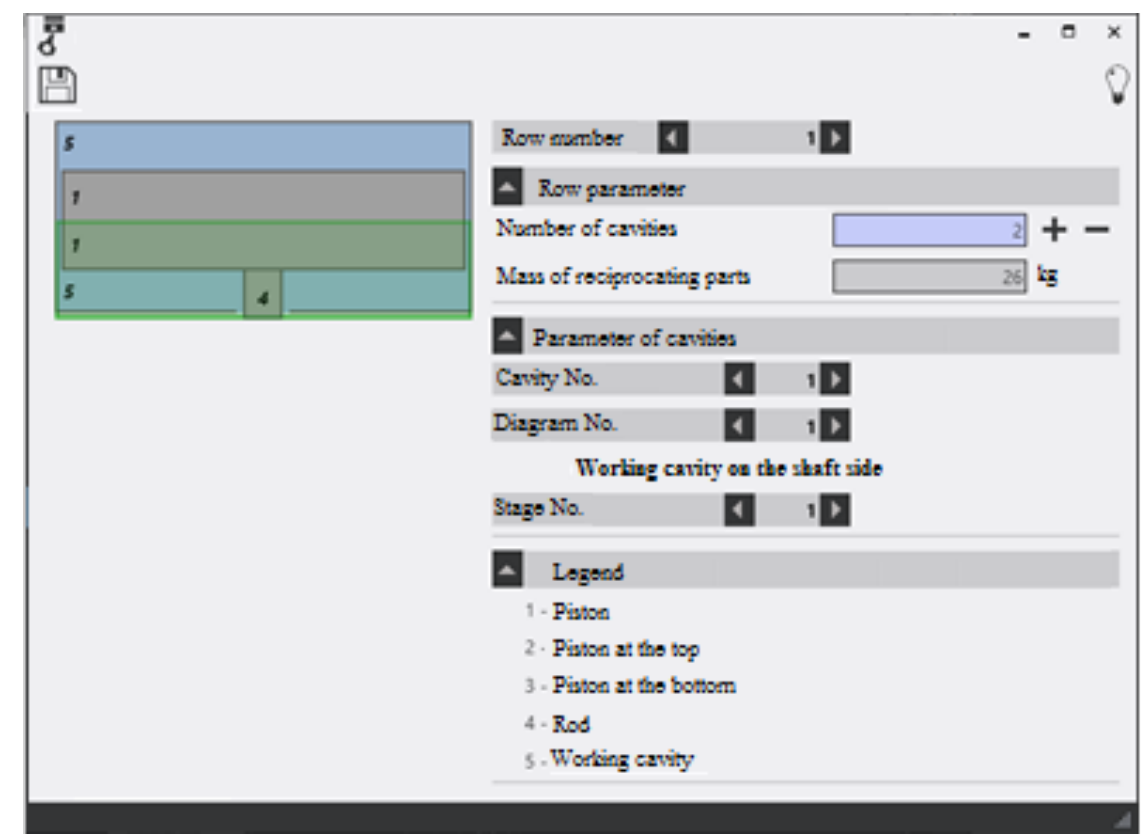

Figure 4: Row Constructor.

Figure 5 shows the structural circuit of the motion mechanism and the initial arrangement of stages by rows. The compressor has four rows located at an angle of 90 degrees to each other. The compressor piston rods are connected to the common throw. The compressor under consideration has four single-action stages each of which is located in an individual row. 


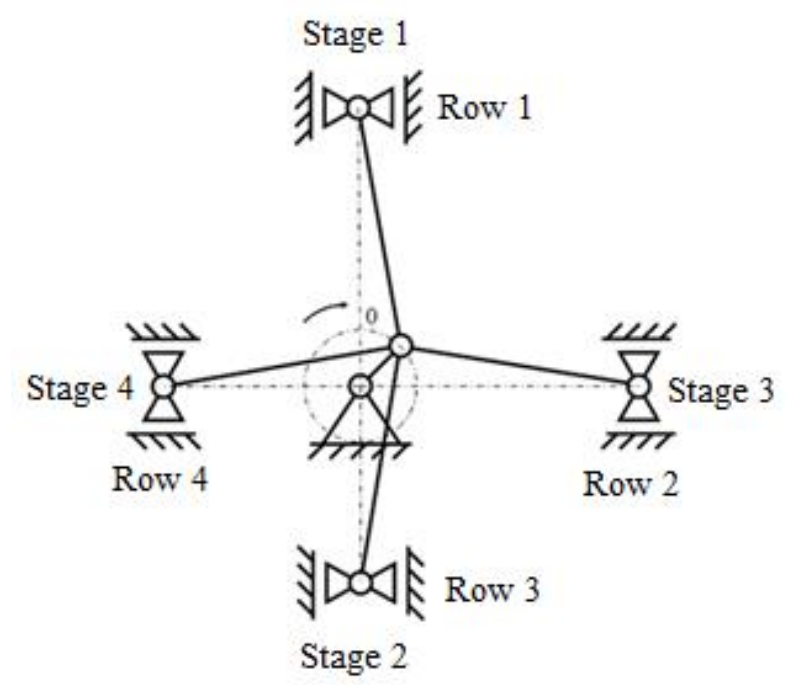

Figure 5: Diagram of Motion Mechanism.

In the frames of this study it was necessary to obtain the compressor torque diagram which does not change its sign during a single turn of the crankshaft. The calculation study was carried out in two stages. At the first stage, the influence on the torque diagram was made by changing the parameters of stage and their location without structural alterations; at the second stage, the best torque diagram was selected due to structural alteration of the compressor with the technical characteristics unchanged.

\section{CALCULATION RESULTS AND THEIR DISCUSSION}

Let us consider the torque diagram (see Figure 6) for the compressor initial design. With reference to the given diagram it can be seen that at the set parameters the constant-sign condition of the torque is not provided. The torque sign change is connected with that the gas load is not equal by the rows. The reason lies in that all stages are of a single action, so the load drop by stages will be different due to the force on the crankcase side. In this case the transition to the negative area is caused by the first stage. This can be seen on the diagram by the torque curves which are given for each row individually (see Figure 6).

Irregularity of torque change (see Figure 6) is due to peculiarities of the working process behavior in the compressor. Gas forces acting to the piston, as well as inertia forces from reciprocating masses are not constant and change depending on the crankshaft angle. Figure 7 shows the diagram of forces acting in the compressor first row.

Torque values less than the average one will indicate that at this moment the engine gives more energy than necessary. And vice versa, the values more than the average one will indicate that at this moment lack of energy from the engine takes place. The average torque is a conditional-constant torque which makes the work during a single turn of the crankshaft same as the actual compressor [6]. A flywheel is used to smooth the angular speed irregularity. It is intended to maintain the shaft angular speed oscillation within the given irregularity through accumulation or giving-up of energy. More uniform torque diagram makes it possible to decrease the flywheel weight. 


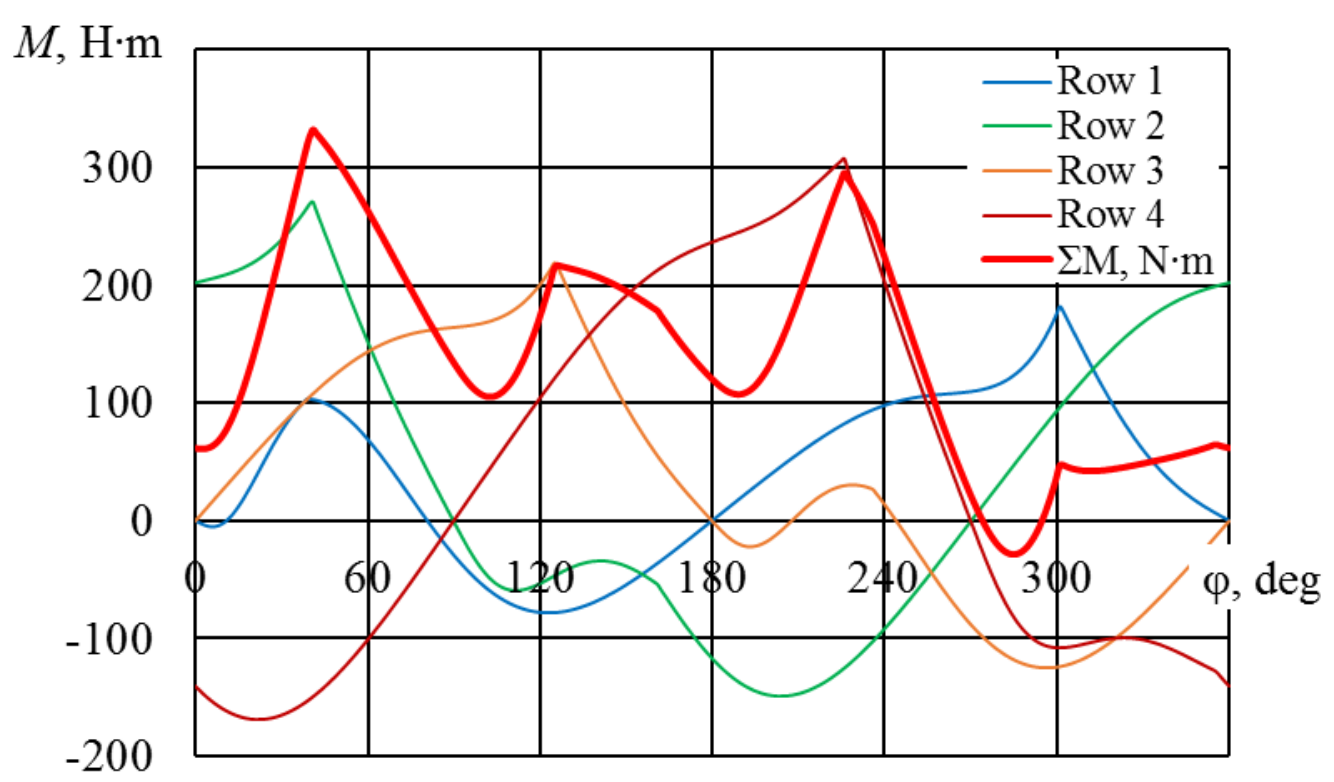

Figure 6: Torque Diagram of the Compressor Initial Design.

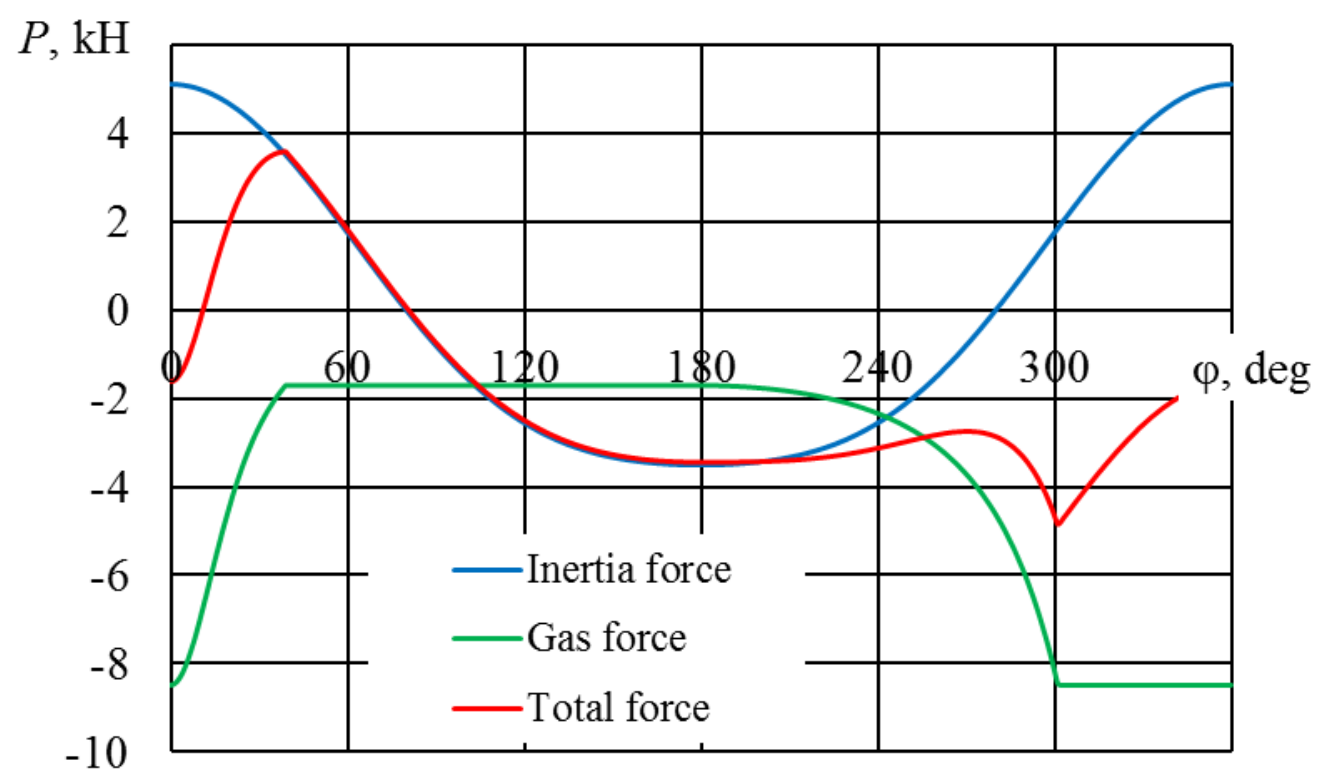

Figure 7: Diagram of First Row Forces.

So, the task consists in obtaining the most uniform torque diagram which has a constant sign during a single revolution of the crankshaft. Influence on the torque diagram is possible by several methods: change in the stages arrangement by the compressor rows (variant 1); change of the dead space by compressor stages (variant 2); change in the compressor piston diameters (variant 3); change of the compressor design (variant 4).

Let us consider the influence of different variants on the torque diagram. Figure 8 gives a comparison of torque curves for variant 1 and variant 2 with the initial design. 


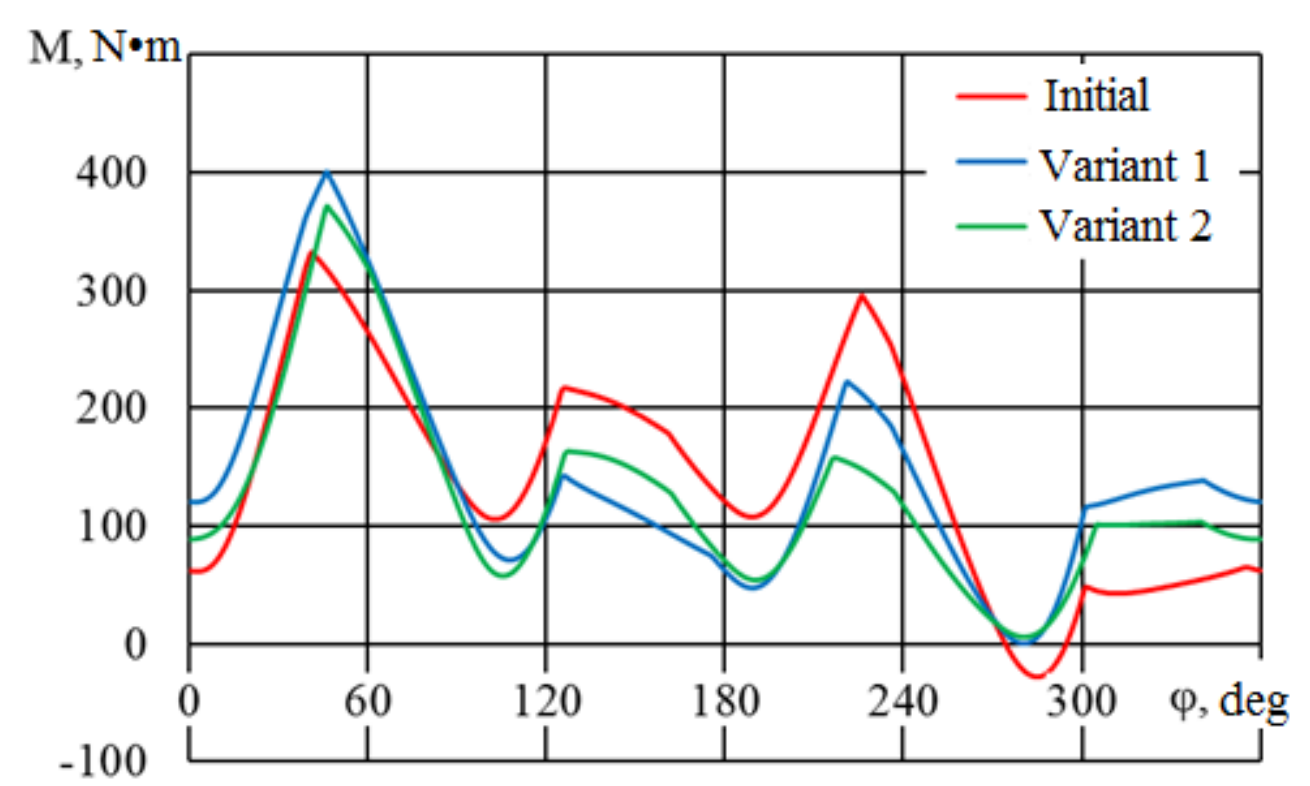

Figure 8: Torque Curves for Different Variants.

Change in the stage sequence (variant 1) from 1-3-2-4 (see Figure 5) to 1-4-2-3 made it possible to slightly approach to the positive area, however, there is no necessary margin of the compressor stable operation. The torque curve became more irregular and has one apparent peak (forth stage influence) at the beginning of the diagram.

A similar picture is observed at change in the dead space by stages (variant 2). In order to make the torque curve smoother it is necessary to increase the dead space at the first stage, which shall lead to decrease in the compressor output. Or, it is necessary to decrease the dead space at the fourth stage, which is not possible due to structural peculiarities of the stage (small sizes). Besides, this shall lead to increase in the ratio between pressures in the fourth stages and, consequently, to increase of the final temperature. Figure 8 shows a diagram for the best variant of the dead spaces distribution. It is seen from the figure that though the torque diagram lies in the positive area, the compressor stable operation margin is very small. Influence of the piston diameter change (variant 3) is similar to the influence of the dead space change (variant 2), since in both cases the influence on the gas force is made by redistribution of pressures between the stages. It is necessary to have in view that the first stage diameter must not be decreased, since this will lead to decrease in the compressor output. Significant decrease in the stage diameters will lead to increase in the ratio between pressures at the previous stages, which shall lead to more heating of gas.

The main problem at selection of rational constant-sign torque diagram is that the gas forces are not uniformly distributed by stages. In order to balance the gas forces by rows it was suggested to change the compressor design (variant 4) which consists in application of differential piston in which the first and the second stage will be located (see Figure 9b). Besides, it was decided to divide these staged by two rows (see Figure 9a). 


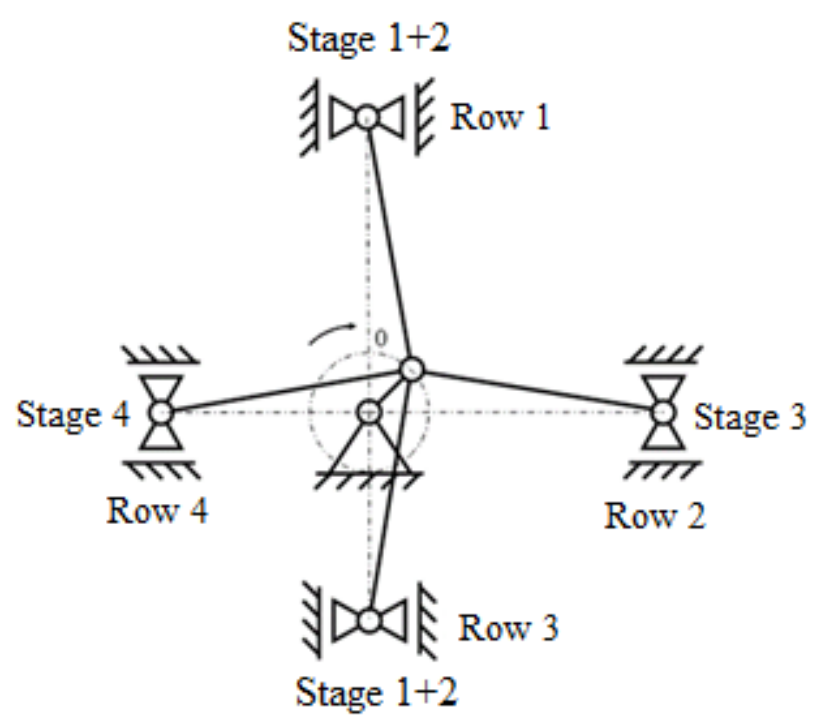

a)

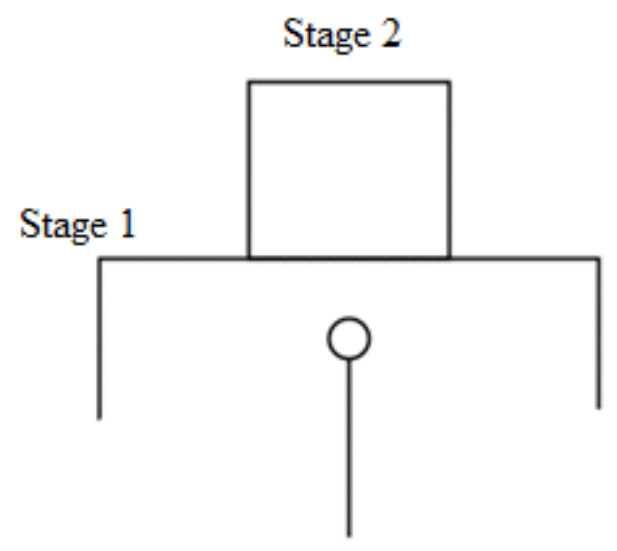

b)

Figure 9. Arrangement of Stages by the Compressor Rows (a) and Differential Piston of the First and Second Stages (b).

The torque diagram of the compressor new design is given in Figure 10.

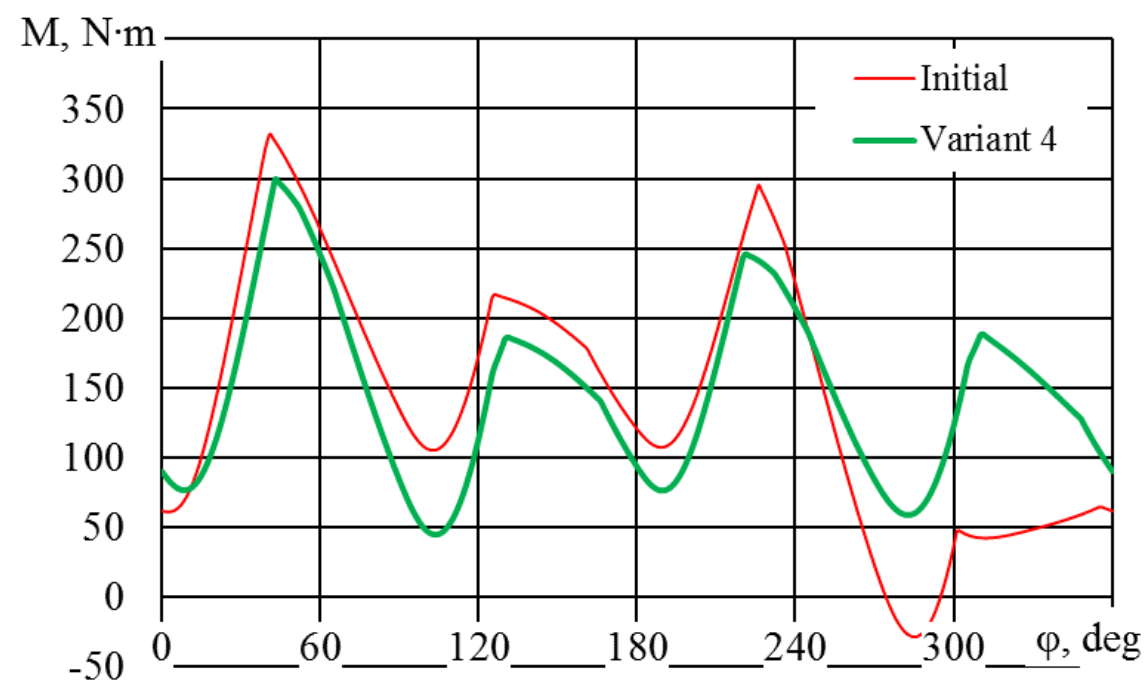

Figure 10: Torque Diagram of the Compressor New Design.

It is seen from the figure that the torque diagram became more uniform and lies completely in the positive area with a high margin of the compressor stable operation. The division of the first and second stages by two rows made it possible to decrease their diameters. The force load to the compressor first and third rows became equal. Considered as disadvantages may be some complication in the design and a necessity to manufacture the differential piston cylinders instead of trunk piston cylinders.

\section{CONCLUSIONS}

The investigation performed shows that the compressor design has an influence on the torque diagram both quantitatively and qualitatively. Change in the design made it possible to significantly improve the compressor torque diagram, which shall lead not only to decrease in the low-frequency vibration, but also to decrease in the flywheel weight. The developed 
calculation program allows analyzing different variants of the compressor design and selecting the most rational one as early as at engineering stage.

\section{REFERENCES}

1. Frenkel M.I. Reciprocating compressors. L.: Mashinostroyeniye, 1969. 740 p. Russian.

2. Chetvertakov V.A. On the balance of the variable overturning moment of piston units. Sudostroyeniye, 1984. №12. Russian.

3. Kotlov A.A., Kuznetsov L.G. and Burakov A.V. Analysis of Reciprocating Booster Compressor Performance by Mathematical Modelling Methods. - AIP Conference Proceedings 2141, 030012 (2019); https://doi.org/10.1063/1.5122062.

4. Istomin P. A. Dynamics of internal combustion engines. L.: Sudostroyeniye, 1966. 280 p.

5. Fotin B.S. Reciprocating compressors / Pod obshch. red. B.S. Fotina. L.: Mashinostroyeniye, 1987. 372 p. Russian.

6. Plastinin P.I. Reciprocating compressors. Tom 2. Structural Design Basics. 3 ed. M.: KolosS, 2008. 711 p. Russian.

7. A.A. Kotlov. Mathematical model of MP pneumatic reciprocating compressor for solution of energy audit tasks: thesis Candidate of Technical Sciences, SPb. 2011. 138 p. Russian.

8. Weifeng $W u$, Zhao Zhang and Quanke Feng. Investigation on the vibration balance approach for four-row star type reciprocating compressors.

9. Kotlov A.A. Research of Two-Stage Reciprocating Compressor by Methods of Physical and Mathematical Experiments. - AIP Conference Proceedings 2141, 030021 (2019); https://doi.org/10.1063/1.5122071.

10. K. M. Ignatiev, B. S. Chrustalev, M. M. Perevozchikov and V. B. Zdaslinsky, "Simulation and parametric analysis of the suction valve and muffler off small reciprocating compressor," in Proceedings of the 1996 International Comperssor Engineering Conference at Purdue, 1 (W. Laafayette, USA, 1996), pp. 201-210.

11. B. S. Chrustalev, A. G. Krasnikov and N. V. Adaev, "Analysis of nonstationary processes of heat and mass exchange in the piston compressor suction system for natural gas compression," in Proceedings of the 1998 International Comperssor Engineering Conference at Purdue, (W. Laafayette, USA, 1998), pp. 543-549.

12. Isakov, V. P. Automatic valves of piston compressors for various ranges of application. Chemical and petroleum engineering / V. P. Isakov, B. S. Chrustalev. Vol.31. №11-12. 1995; July 1996 USA. NewYork.

13. Lohn, Sérgio Koerich and Pereira, Evandro Luiz Lange, "Numerical Investigation of the Gas Leakage through the PistonCylinder Clearance of Reciprocating Compressors" (2014). International Compressor Engineering Conference. Paper 2295.

14. Sanvezzo Jr, Joel and Deschamps, Cesar J., "A Heat Transfer Model Combining Differential and Integral Formulations for Thermal Analysis of Reciprocating Compressors" (2012). International Compressor Engineering Conference. Paper 2104.

15. Soedel W. Introduction of computer simulation of positive displacement type compressors. Purdue University School of Mechanical Engineering, West Lafayette, Indiana, 47907.

16. A. A. Kotlov, I. A. Maksimenko and Y. L. Kuznetsov. The influence of profile geometric parameters on characteristics of rotorgearing compressor. IOP Conf. Series: Materials Science and Engineering 425 (2018) 012012 doi:10.1088/1757$899 X / 425 / 1 / 012012$.

17. A. A. Kotlov. Influence of suction port parameters on integral characteristics of screw-type compressor. IOP Conf. Series: Materials Science and Engineering 425 (2018) 012014 doi:10.1088/1757-899X/425/1/012014. 
18. Galerkin Y., Soldatova K., Drozdov A. Centrifugal compressor efficiency types and rational application. International Conference on Compressors and their Systems. London. City University. - UK. -2013. - P. 533-542

19. Galerkin Y., Drozdov A. Sample of CFD optimization of a ctentrifugal compressor stage. International Conference on Compressors and their Systems. London. City University. - UK. -2015. - http://iopscience.iop.org/article/10.1088/1757899X/90/1/012040/meta. DOI: 10.1088/1757-899X/90/1/012041.

20. Galerkin Y., Drozdov A. New generation of Universal modeling for centrifugal compressors calculation. International Conference on Compressors and their Systems. London. City University. - UK. - $2015 . \quad$ http://iopscience.iop.org/article/10.1088/1757-899X/90/1/012041/meta. DOI: 10.1088/1757-899X/90/1/012040.

21. Galerkin Y., Drozdov A. Centrifugal compressor stage design principlies cheking. ASME Gas Turbine India Conference. 2015. - GTINDIA2015-1213. - http://www.asmeconferences.org/GTIndia2015/PaperAccepted.cfm?noToolbar=yes. DOI: 10.1115/GTINDIA2015-1213.

22. Y. Galerkin, L. Marenina, Soldatova K. CFD wind tunnel tests of Centrifugal stage return channel vane cascades. ASME Gas $\begin{array}{llll}\text { Turbine } \quad \text { India } \quad \text { Conference. } & - & 2015 . & \text { G }\end{array}$ http://www.asmeconferences.org/GTIndia2015/PaperAccepted.cfm?noToolbar=yes. DOI: 10.1115/GTINDIA2015-1216.

23. Galerkin Y., Voinov I., Drozdov A. Comparison of CFD-calculations of centrifugal compressor stages by NUMECA Fine/Turbo and ANSYS CFX programs. International Conference on Compressors and their Systems. London. City University. - UK. - 2017. - http://iopscience.iop.org/article/10.1088/1757-899X/232/1/012046. - DOI: 10.1088/1757-899X/232/1/012044.

24. Galerkin Y., Drozdov A. The numerical study of the rake angle of impeller blade in centrifugal compressor. International Conference on Compressors and their Systems. London. City University. - UK. - $2017 . \quad$ http://iopscience. iop.org/article/10.1088/1757-899X/232/1/012046. - DOI: 10.1088/1757-899X/232/1/012036.

25. Galerkin, Y. The application of mathematical models for industrial centrifugal compressor optimal design / Y. Galerkin, K. Soldatova, A. Drozdov // ICCMS 2018: 10th International Conference on Computer Modeling and Simulation. - Australia. Sydney. -Paper ID: 008. - 2018. P. 187-191

26. Drozdov A., Galerkin Y. Modeling the non-incidence inlet flow rate coefficient in a centrifugal compressor impeller. - Oil and Gas Engineering (OGE-2018). - AIP Conf. Proc. 2007. - Omsk. - Russia. - 2018. - 030052-1-030052-10; https://doi.org/10.1063/1.5051913

27. Rekstin A.F., Drozdov A.A., Solovyeva O.A., Galerkin Y.B. Two mathematical models centrifugal compressor stage vaneless diffuser comparison. - Oil and Gas Engineering (OGE-2018). -AIP Conf. Proc. 2007. - Omsk. - Russia. - 2018. - 030035-1030035-12; https://doi.org/10.1063/1.5051896

28. Drozdov A.A., Yaroshenko V.I., Pisarev Y.N. The geometric model of the multistage centrifugal compressor flow path in the ANSYS CFX. - Oil and Gas Engineering- AIP Conf. Proc. 2141. - Omsk. - Russia. - 2019, 030017-030017-12, https://doi.org/10.1063/1.5122067

29. Galerkin Y., Drozdov A., Solovyeva O. Vaneless diffuser for low flow rate centrifugal compressor stage. - Proceedings of 13th European Conference on Turbomachinery Fluid dynamics \& Thermodynamics. - Paper ID: ETC2019-329. - ETC13, Lausanne, Switzerland. - April 8-12, 2019

30. Galerkin Y., Drozdov A., Solovyeva O. and Kabalyk K. Development of mathematical model of Universal modeling method for centrifugal compressors calculation. - E3S Web of Conferences 140, 06002 (2019). - International Scientific Conference on Energy, Environmental and Construction Engineering (EECE-2019) https://doi.org/10.1051/e3sconf/201914006002 